\title{
The distance to the LMC cluster NGC 1866; clues from the cluster Cepheid population
}

\author{
M. A. T. Groenewegen ${ }^{1}$ and M. Salaris ${ }^{2}$ \\ 1 Instituut voor Sterrenkunde, PACS-ICC, Celestijnenlaan 200B, 3001 Leuven, Belgium \\ 2 Astrophysics Research Institute, Liverpool John Moores University, Twelve Quays House, Birkenhead CH41 1LD, UK
}

Received 18 June 2003 / Accepted 25 August 2003

\begin{abstract}
Recent investigations aimed at estimating the distance to the young LMC cluster NGC 1866 have made use of Red Clump stars in the surrounding LMC field, together with empirical and theoretical Main-Sequence fitting methods, and have found significantly different distances for the field and the cluster, the latter being closer by in distance modulus by $\Delta(D M) \sim$ 0.20 mag. In this paper we (re-)consider the Cepheid star population of NGC 1866, to try to shed some light on this discrepancy. By combining various extensive photometric datasets in $B, V, I$ and single-epoch 2MASs $J H K$ photometry, $P L$ relationships for the cluster Cepheids are obtained. A comparison between the field LMC and cluster $P L$ relationships for the reddening free Wesenheit index gives a firm determination of the distance between the cluster and the LMC main body ( 0.04 mag in distance modulus, the cluster being more distant) which, coupled to a model for the geometry of the LMC disk, provides $\Delta(D M)$ ranging between 0.0 and $-0.11 \mathrm{mag}$. The simultaneous comparison of the $P L$ relationships in $B, V$ and $I$ for the cluster and LMC field gives an estimate of the cluster reddening, which results to be $E(B-V)=0.12 \pm 0.02$. This determination is higher than the canonical value of 0.06 mag used in all previous studies, but we show that it is not in contradiction with a re-analysis of independent estimates. The adoption of the LMC extinction law recently presented by Gordon et al. (2003) does not change these results. The cluster Main Sequence fitting distance obtained with this new reddening is $D M=18.58 \pm$ 0.08 , fully compatible with the Red Clump value of $D M=18.53 \pm 0.07$ (random) ${ }_{-0.05}^{+0.02}$ (systematic) and the Cepheid constraint on $\Delta(D M)$. Finally, we determined the distance to the cluster by using a Cepheid Wesenheit $P L$ relationship with slope coming from LMC observations, and absolute magnitude zero point calibrated on Hipparcos parallaxes of Galactic Cepheids, in the assumption that the relationship is independent of metallicity; the resulting $D M=18.65 \pm 0.10$ is not an accurate estimate of the LMC distance because of possible metallicity effects but, when compared to the revised Main Sequence fitting value, it points out to a possibly weak dependence of the Wesenheit $P L$ relationship on the Cepheid chemical composition, at least in the period range between 2.5 and 3.5 days.
\end{abstract}

Key words. stars: distances - Cepheids - Magellanic Clouds - distance scale

\section{Introduction}

Two recent papers considered the distance to the young LMC cluster NGC 1866 based on empirical and theoretical Main-Sequence (MS) fitting techniques, and on the Red Clump (RC) distance to the LMC field around the cluster. Walker et al. (2002) used a MS-fitting method employing theoretical isochrones to derive a distance modulus $(D M)$ to the cluster $D M=18.35 \pm 0.05$, and a reddening of $E(B-V)=$ 0.060-0.064. Salaris et al. (2003a, hereafter S03) employed the same cluster photometry to derive $D M=18.33 \pm 0.08$ (for an adopted reddening of 0.064) using a completely empirical MS-fitting method, based a large sample of local subdwarfs with accurate parallax and $[\mathrm{Fe} / \mathrm{H}]$ determination; however, when they applied the RC method (following the procedure by Alves et al. 2002 and the population corrections

Send offprint requests to: M. Groenewegen,

e-mail: groen@ster.kuleuven.ac.be by Salaris \& Girardi 2002) for deriving the distance to the surrounding LMC field, it resulted a distance modulus $D M=$ $18.53 \pm 0.07$ (a similar value of $18.47 \pm 0.05$ is obtained by Pietrzyński \& Gieren 2002 using the RC in the $K$-band). This $D M$ discrepancy $\Delta(D M)=0.20 \pm 0.10$ between $\mathrm{RC}$ and MS-fitting distances reflects the more general dichotomy in the LMC distance estimates found in the literature (see, e.g., Benedict et al. 2002 for a recent summary of the LMC distance determinations). S03 discussed possible reasons for this occurrence, as an underestimated cluster metallicity, a photometric zero point error, the possibility that the cluster is located about $5 \mathrm{Kpc}$ closer than the underlying field population, but no definitive conclusion was reached.

NGC 1866 contains a sizable Cepheid population, and therefore an independent distance estimate is potentially available. Shapley \& Nail (1950) and Thackeray (1951) independently discovered the first Cepheids in NGC 1866, and first 
photometry and periods were published by Arp \& Thackeray (1967; hereafter AT67); from Period-Luminosity $(P L)$ and Period-Luminosity-Colour $(P L C)$ relations with the then available calibration they derived a $D M$ to the cluster of $18.44 \pm$ 0.15 (for a reddening of $E(B-V)=0.06$ ). After the publication by Walker (1987, hereafter Wa87) of the first CCD light curves for seven cluster Cepheids, Storm et al. (1988) report the discovery of 10 new Cepheid candidates. Welch et al. (1991; hereafter We91) present new CCD photometry and radial velocity measurements of these new and previously known objects. For a reddening of $E(B-V)=0.06$, and an assumed $P L C$-relation they derive $D M=18.57 \pm 0.01$ (internal error only). These data were then used by Côte et al. (1991) for a Baade-Wesselink analysis to derive effective temperatures and radii, and $D M=$ $18.6 \pm 0.3$. Gieren et al. (1994) derived an improved $D M=$ $18.47 \pm 0.20$ from the first VRI-based Baade-Wesselink analysis of 3 cluster Cepheids. Gieren et al. (2000a; hereafter G2000) report extensive new $B V R I$ photometry for seven Cepheids and provide improved periods, and at the same time Gieren et al. (2000b) discuss the application of the infrared surface brightness Baade-Wesselink method on a Cepheid (HV 12198) in the cluster. From this one star, they determine $D M=18.42 \pm 0.10$.

It is clear that today extensive photometry for many of the Cepheids in NGC 1866 does exist. Single-epoch infrared data are also potentially available from the 2MAss survey. Current Cepheid-based distance estimates date back to more than 10 years ago, i.e. in the era before the Hipparcos based calibration of the $\mathrm{ZP}$ of the Galactic $P L$-relation, and before the huge datasets of field Cepheids in the Magellanic Clouds, discovered by the microlensing surveys. The aim of the present paper is therefore to combine all available data for the Cepheids in NGC 1866 with our current knowledge of field LMC and Galactic Cepheids, in order to shed some light on the dichotomy of distances estimated from MS-fitting and the RC-method. In Sect. 2 we discuss the available Cepheid photometric data; inferences from their $P L$ relationships will be analysed in Sect. 3, and a discussion about the implications for the cluster distance follows in Sect. 4.

\section{Photometry of NGC 1866 Cepheids}

We considered the photometry ( $V$, and when available $B$ and $I$ ) from G2000, We91, Wa87 and AT67. As previously noted and discussed in the relevant papers, there is for some stars a small difference between the different sets of photometry (e.g., see the phase diagrams by G2000). Furthermore, the photometry in AT67 is made of photographic magnitudes whereas data in the other papers are obtained using CCDs. Lastly, G2000 and We91 quote (internal) errors while Wa87 and AT67 do not. On the other hand we want to use as much data as possible with representative errors to make full use of all available information. It should also pointed out that crowding is a potential problem for the Cepheids closer to the centre of the cluster (in particular the Cepheids with the $V$ prefix in their name) and new photometry under excellent seeing conditions would be valuable.

In order to do so we took a two-step approach. As a first step, we fixed the periods to the most accurate known values (i.e. as quoted in the paper based on the single dataset with most observations, hence G2000 in most cases). For each dataset we then solved for the amplitude and phase, using the numerical code "Period98" (Sperl 1998). We also considered lightcurves with a suitable number of harmonics (typically 3 to 5), solving again for the amplitudes and phases, the relevant output quantities being the mean magnitude and the rms. The difference between the mean magnitudes obtained with different datasets gives an indication of the photometric offsets, and the rms gives an indication of the error in an individual measurement.

Based on this exercise, an error of 0.008 mag was assigned to Wa87 data, and 0.05 to the $V$-band data in AT67. In addition, the following offsets were added to the published photometries in order to put them on the same system as G2000: +0.039 mag ( $I$, Wa87, HV 12197), +0.082 mag ( $V$, We91, HV 12200), -0.066 mag (I, Wa87, HV 12203), and to the photographic $V$-band in AT67 we added +0.095 mag for HV 12197, 12198 , 12199, 12205, +0.212 mag for HV 12202 and +0.079 mag for HV 12203.

In case of the $B$-band we added $+0.04 \mathrm{mag}$ (Wa87, HV 12197), -0.06 mag (We91, HV 12197) +0.02 mag (Wa87, HV 12199), +0.02 mag (We91, HV 12199), +0.15 mag (We91, HV 12200), -0.02 mag (Wa87, HV 12202), +0.05 mag (We91, HV 12202), +0.06 mag (Wa87, HV 12203), +0.03 mag (We91, HV 12203) and -0.02 mag (We91, V7).

HV 12204 was not considered as it is likely a non-member based on its radial velocity (Wa87).

In a second step we combined all datasets (with offsets and proper weighting applied) and performed a Fourier analysis solving for the primary frequency, amplitudes and phases. The results are listed in Tables $1-3$. When fitting the $B$ and $I$-band lightcurve the frequencies where fixed to that determined from the $V$-lightcurves. Also listed is the rms in the final fit. This number will be used in the next section to characterise the error in the mean magnitude when using the $P L$-relationship.

In the case of the $B$-band, keeping the frequency also as a free parameter resulted in a different frequency by $(1-3) \times 10^{-6}$ cycles/day at most, and no significant change in the mean magnitude and rms values. For the stars in common, the periods derived here and those quoted in G2000 agree within their respective $2 \sigma$ error bars.

The quantities $R_{21}=A_{2} / A_{1}$ and $\phi_{21}=\phi_{2}-2 \phi_{1}$ (where $A_{i}$ and $\phi_{i}$ represent the amplitude and phase of the $(i-1)$-harmonic in the Fourier expansion) can be used to distinguish fundamental (FU) from first overtone (FO) pulsators (e.g. Udalski et al. 1999b); when applying this technique we found that Cepheid V8 is a first overtone pulsator (We87 already suggested this purely on the basis of its short period). For V4 the situation is ambiguous and it is kept as a FU pulsator. In case of V6, although the amplitude ratio suggests it is a FU, the object is treated as a FO pulsator for reasons given below (note that We87 also suggested it to be a probable FO pulsator). When plotted on a $V$-band $P L$-diagram (like Fig. 3 below), it would stand out as a clear outlier at its observed period of 2.05 days, being about 0.4 mag brighter than the mean relation at that period, a deviation by many sigmas. At the same time, it falls almost exactly in the middle of datapoints 
Table 1. Fourier decomposition of $V$-lightcurves.

\begin{tabular}{|c|c|c|c|c|c|c|}
\hline Name & $<V>$ & Period $(\mathrm{d})$ & $\mathrm{rms}$ & $N$ & Ampl & Phase \\
\hline \multirow[t]{4}{*}{12197} & 16.102 & $3.14374(3)$ & 0.028 & 100 & 0.226 & 0.399 \\
\hline & & & & & 0.079 & 0.208 \\
\hline & & & & & 0.0305 & 0.984 \\
\hline & & & & & 0.0137 & 0.736 \\
\hline \multirow[t]{4}{*}{12198} & 15.976 & $3.52275(3)$ & 0.030 & 134 & 0.258 & 0.418 \\
\hline & & & & & 0.112 & 0.260 \\
\hline & & & & & 0.0553 & 0.113 \\
\hline & & & & & 0.0162 & 0.021 \\
\hline \multirow[t]{5}{*}{12199} & 16.289 & $2.639166(2)$ & 0.059 & 113 & 0.270 & 0.963 \\
\hline & & & & & 0.127 & 0.330 \\
\hline & & & & & 0.0610 & 0.735 \\
\hline & & & & & 0.0349 & 0.087 \\
\hline & & & & & 0.0093 & 0.439 \\
\hline \multirow[t]{5}{*}{12200} & 16.247 & $2.72499(3)$ & 0.042 & 63 & 0.325 & 0.767 \\
\hline & & & & & 0.153 & 0.927 \\
\hline & & & & & 0.0807 & 0.103 \\
\hline & & & & & 0.0599 & 0.316 \\
\hline & & & & & 0.0258 & 0.417 \\
\hline \multirow[t]{4}{*}{12202} & 16.080 & $3.10118(1)$ & 0.037 & 122 & 0.211 & 0.691 \\
\hline & & & & & 0.0903 & 0.786 \\
\hline & & & & & 0.0349 & 0.914 \\
\hline & & & & & 0.0091 & 0.897 \\
\hline \multirow[t]{5}{*}{12203} & 16.140 & $2.95414(2)$ & 0.028 & 115 & 0.252 & 0.962 \\
\hline & & & & & 0.106 & 0.340 \\
\hline & & & & & 0.0519 & 0.734 \\
\hline & & & & & 0.0232 & 0.119 \\
\hline & & & & & 0.0054 & 0.606 \\
\hline \multirow[t]{3}{*}{12205} & 15.965 & $3.21048(2)$ & 0.090 & 32 & 0.330 & 0.728 \\
\hline & & & & & 0.143 & 0.862 \\
\hline & & & & & 0.122 & 0.117 \\
\hline \multirow[t]{2}{*}{ V4 } & 16.068 & $3.31886(2)$ & 0.022 & 48 & 0.113 & 0.391 \\
\hline & & & & & 0.027 & 0.185 \\
\hline \multirow[t]{4}{*}{ V6 } & 16.111 & $2.05442(2)$ & 0.030 & 36 & 0.080 & 0.207 \\
\hline & & & & & 0.049 & 0.049 \\
\hline & & & & & 0.049 & 0.203 \\
\hline & & & & & 0.019 & 0.132 \\
\hline \multirow[t]{3}{*}{ V7 } & 15.973 & $3.38827(2)$ & 0.065 & 79 & 0.131 & 0.723 \\
\hline & & & & & 0.0364 & 0.827 \\
\hline & & & & & 0.0267 & 0.912 \\
\hline \multirow[t]{4}{*}{ V8 } & 16.159 & $2.05249(2)$ & 0.036 & 46 & 0.128 & 0.033 \\
\hline & & & & & 0.047 & 0.624 \\
\hline & & & & & 0.021 & 0.898 \\
\hline & & & & & 0.016 & 0.584 \\
\hline
\end{tabular}

Listed are the identifier, mean magnitude, period in days with the uncertainty in the last digit between parenthesis, the rms in the fit, the number of datapoints, and then the amplitude and phase of the Fourier components, one component in each line. The solutions listed for $B$ and $I$ in Tables 2 and 3 have been obtained with the frequency indicated here. Leaving the frequency as a free parameter will lead to very small differences in the derived quantities, that have been the basis for attributing an error to the period.

of FO pulsators in the LMC field at that period (Udalski et al. 1999a). In addition, the number of field LMC Cepheids in the period range $2.03<P<2.07 \mathrm{~d}$ is $0 \mathrm{FU}$ and $15 \mathrm{FO}$, and in the extended range $2.00<P<2.10 \mathrm{~d}$ is $4 \mathrm{FU}$ and $33 \mathrm{FO}$.
Table 2. Fourier decomposition of $B$-lightcurves.

\begin{tabular}{|c|c|c|c|c|c|}
\hline Name & $<B>$ & $\mathrm{rms}$ & $N$ & Ampl & Phase \\
\hline \multirow[t]{4}{*}{12197} & 16.741 & 0.024 & 50 & 0.338 & 0.418 \\
\hline & & & & 0.114 & 0.222 \\
\hline & & & & 0.0545 & 0.041 \\
\hline & & & & 0.0088 & 0.802 \\
\hline \multirow[t]{4}{*}{12198} & 16.627 & 0.024 & 83 & 0.284 & 0.384 \\
\hline & & & & 0.159 & 0.272 \\
\hline & & & & 0.0790 & 0.138 \\
\hline & & & & 0.0209 & 0.041 \\
\hline \multirow[t]{5}{*}{12199} & 16.916 & 0.015 & 62 & 0.411 & 0.974 \\
\hline & & & & 0.177 & 0.322 \\
\hline & & & & 0.0833 & 0.723 \\
\hline & & & & 0.0454 & 0.061 \\
\hline & & & & 0.0153 & 0.4458 \\
\hline \multirow[t]{5}{*}{12200} & 16.913 & 0.053 & 62 & 0.518 & 0.779 \\
\hline & & & & 0.230 & 0.929 \\
\hline & & & & 0.137 & 0.084 \\
\hline & & & & 0.0594 & 0.299 \\
\hline & & & & 0.0323 & 0.389 \\
\hline \multirow[t]{4}{*}{12202} & 16.756 & 0.019 & 71 & 0.303 & 0.706 \\
\hline & & & & 0.104 & 0.798 \\
\hline & & & & 0.0442 & 0.912 \\
\hline & & & & 0.0114 & 0.163 \\
\hline \multirow[t]{5}{*}{12203} & 16.811 & 0.026 & 64 & 0.378 & 0.976 \\
\hline & & & & 0.150 & 0.349 \\
\hline & & & & 0.0919 & 0.723 \\
\hline & & & & 0.0235 & 0.102 \\
\hline & & & & 0.0195 & 0.608 \\
\hline \multirow[t]{3}{*}{12205} & 16.572 & 0.013 & 11 & 0.557 & 0.748 \\
\hline & & & & 0.222 & 0.836 \\
\hline & & & & 0.145 & 0.982 \\
\hline \multirow[t]{2}{*}{ V4 } & 16.715 & 0.034 & 45 & 0.177 & 0.415 \\
\hline & & & & 0.038 & 0.218 \\
\hline \multirow[t]{3}{*}{ V6 } & 16.696 & 0.123 & 24 & 0.079 & 0.194 \\
\hline & & & & 0.034 & 0.904 \\
\hline & & & & 0.091 & 0.214 \\
\hline \multirow[t]{3}{*}{ V7 } & 16.622 & 0.028 & 47 & 0.205 & 0.718 \\
\hline & & & & 0.0664 & 0.805 \\
\hline & & & & 0.0344 & 0.964 \\
\hline \multirow[t]{3}{*}{ V8 } & 16.773 & 0.043 & 46 & 0.205 & 0.050 \\
\hline & & & & 0.045 & 0.603 \\
\hline & & & & 0.031 & 0.905 \\
\hline
\end{tabular}

This statistical argument (in the hypothesis that cluster and field Cepheids share the same properties), and the fact that it is located on top of the $V$-band $P L$-relation of LMC field FO Cepheids, make us believe that V6 is an overtone pulsator. If this object is excluded from our analysis, the results we present in the following are completely unaffected. All other Cepheids in our sample appear to be FU pulsators.

For the FO variables V6 and V8 the observed period $\left(P_{1}\right)$ has been transformed into the corresponding fundamental one $\left(P_{0}\right)$ according to (Feast \& Catchpole 1997):

$P_{1} / P_{0}=0.716-0.027 \log P_{1}$. 
Table 3. Fourier decomposition of $I$-lightcurves.

\begin{tabular}{|c|c|c|c|c|c|}
\hline Name & $<I\rangle$ & $\mathrm{rms}$ & $N$ & Ampl & Phase \\
\hline \multirow[t]{3}{*}{12197} & 15.379 & 0.021 & 66 & 0.140 & 0.370 \\
\hline & & & & 0.050 & 0.209 \\
\hline & & & & 0.0059 & 0.958 \\
\hline \multirow[t]{4}{*}{12198} & 15.234 & 0.016 & 74 & 0.156 & 0.374 \\
\hline & & & & 0.067 & 0.248 \\
\hline & & & & 0.036 & 0.149 \\
\hline & & & & 0.018 & 0.998 \\
\hline \multirow[t]{5}{*}{12199} & 15.591 & 0.015 & 67 & 0.168 & 0.963 \\
\hline & & & & 0.081 & 0.325 \\
\hline & & & & 0.042 & 0.741 \\
\hline & & & & 0.020 & 0.047 \\
\hline & & & & 0.0085 & 0.515 \\
\hline \multirow[t]{2}{*}{12200} & 15.593 & 0.104 & 15 & 0.231 & 0.768 \\
\hline & & & & 0.168 & 0.945 \\
\hline \multirow[t]{4}{*}{12202} & 15.383 & 0.050 & 50 & 0.140 & 0.653 \\
\hline & & & & 0.067 & 0.832 \\
\hline & & & & 0.055 & 0.823 \\
\hline & & & & 0.020 & 0.921 \\
\hline \multirow[t]{4}{*}{12203} & 15.441 & 0.025 & 67 & 0.146 & 0.931 \\
\hline & & & & 0.063 & 0.341 \\
\hline & & & & 0.021 & 0.773 \\
\hline & & & & 0.012 & 0.145 \\
\hline \multirow[t]{3}{*}{ V7 } & 15.295 & 0.013 & 18 & 0.125 & 0.645 \\
\hline & & & & 0.055 & 0.591 \\
\hline & & & & 0.028 & 0.886 \\
\hline
\end{tabular}

In addition to $B, V$ and $I$ data, single-epoch $J H K$ photometry was collected from the 2 MASs all-sky release (Cutri et al. 2003). Accurate coordinates are not immediately available in the literature for the Cepheids, and therefore coordinates were retrieved using a FITS image containing the necessary WCS (World Coordinate System) keywords and the finding charts in AT67, Storm et al. (1988) and We91. The photometry with errors and the coordinates, as given by 2 MASs, are listed in Table 4. In general, the stars are faint (for 2MAss) and the error bars are substantial. Monitoring these stars in the infrared with modern instrumentation would be valuable. It is immediately evident that the photometry for HV 12200 is very different from all others. Possibly, we have identified the wrong star, or there is an unnoticed problem with the 2MAss photometry for this object. This star was not used when fitting the $K$-band $P L$-relation.

\section{Analysis}

The previous study of the available Cepheid photometry has provided us with mean $B, V$ magnitudes for 11 objects, $K$ single epoch magnitudes for 10 objects, and $I$ mean magnitudes for 7 objects; from the $V$ and $I$ magnitudes we computed the corresponding values of the reddening independent Wesenheit index $W=I-1.55(V-I)$, like in Udalski et al. (1999a, hereafter U99). In Figs. 1-4 we display the $P L$ relationships for the cluster Cepheids in $V, I, B$ and $W$; we fitted to the data, as customary, a $P L$-relation of the type

$m_{\mathrm{c}}=($ slope $) \times \log P+\mathrm{ZP}$

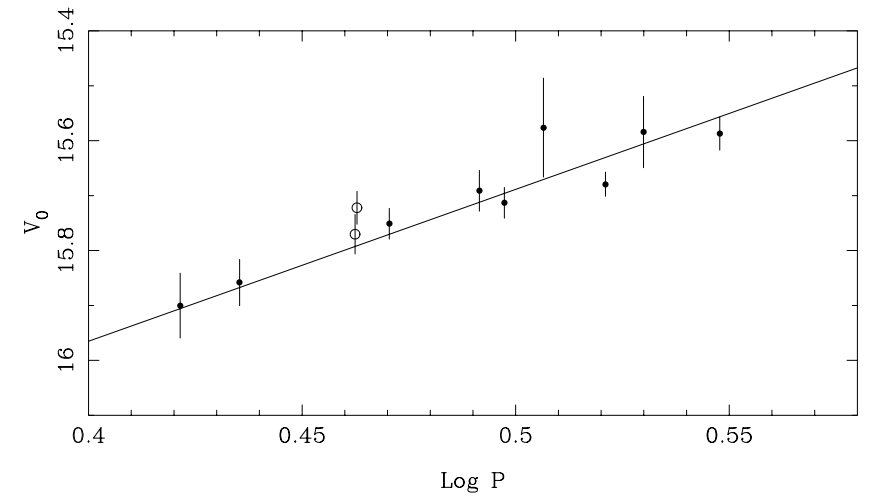

Fig. 1. $P L$ relation in the $V$-band, for the adopted slope of -2.765 and $E(B-V)=0.12$ (see text for a discussion about the cluster reddening). The open circles represent the two overtone pulsators, plotted at their corresponding fundamental period.

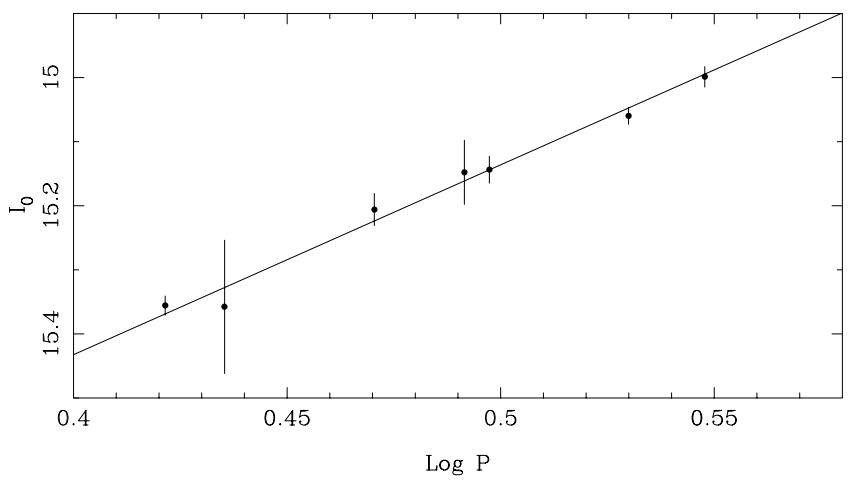

Fig. 2. $P L$ relation in the $I$-band, for the adopted slope of -2.963 and $E(B-V)=0.12$.

with the index $c$ being $W, I, B$ and $V$. We obtained slopes equal to $-3.47 \pm 0.48$ in $W,-2.94 \pm 0.17$ in $I,-2.40 \pm 0.50$ in $B$ and $-2.52 \pm 0.33$ in $V$. Due to the small number of objects the error on the slopes is substantial, but it does still allow interesting inferences. First of all, we have compared these slopes to the corresponding values for the LMC field Cepheids as obtained by Groenewegen (2000) from the data by U99, i.e., $-3.337,-2.963,-2.352^{1}$ and -2.765 in $W, I, B$ and $V$ respectively, with very small errorbars. It is evident that the cluster $P L$ slopes are in formal agreement with the field ones.

We have then compared the cluster slopes with the recent results by Tammann et al. (2003) for Galactic Cepheids; the authors re-calibrated the Galactic $P L$ relationship by combining absolute magnitudes of Cepheids in open clusters (distances obtained from the MS-fitting technique) with absolute magnitudes of other Cepheids obtained from surface brightness methods, and obtained slopes equal to $-2.757 \pm$ $0.112,-3.408 \pm 0.095$ and $-3.141 \pm 0.100$ in $B, I$ and $V$, respectively. These values are significantly different from the results for the field LMC Cepheids, pointing out to a clear dependence of the slopes of the $P L$ relationships on the Cepheid metallicities. It is also evident that the cluster $P L$ slopes are significantly different from the Galactic ones, even accounting

\footnotetext{
${ }^{1}$ For this passband the slope was not given in Groenewegen (2000)
} but has been determined for the present paper in an identical way. 
Table 4. 2MAss photometry.

\begin{tabular}{cccccc}
\hline \hline Id & RA (deg) & Dec $($ deg $)$ & $J$ & $H$ & $K$ \\
\hline 12197 & 78.342284 & -65.503525 & $14.776 \pm 0.037$ & $14.538 \pm 0.068$ & $14.423 \pm 0.099$ \\
12198 & 78.361127 & -65.451439 & $14.816 \pm 0.047$ & $14.490 \pm 0.061$ & $14.469 \pm 0.102$ \\
12199 & 78.354798 & -65.491463 & $14.958 \pm 0.044$ & $14.591 \pm 0.080$ & $14.407 \pm 0.107$ \\
12200 & 78.440586 & -65.459991 & $14.322 \pm 0.071$ & $13.891 \pm 0.079$ & $13.696 \pm 0.094$ \\
12202 & 78.452373 & -65.475998 & $14.638 \pm 0.044$ & $14.297 \pm 0.073$ & $14.153 \pm 0.087$ \\
12203 & 78.459130 & -65.485962 & $14.913 \pm 0.054$ & $14.687 \pm 0.084$ & $14.335 \pm 0.100$ \\
12205 & 78.576648 & -65.510002 & $14.587 \pm 0.040$ & $14.366 \pm 0.056$ & $14.254 \pm 0.066$ \\
V4 & 78.406977 & -65.454140 & $14.746 \pm 0.063$ & $14.406 \pm 0.076$ & $14.520 \pm 0.141$ \\
V6 & 78.424360 & -65.472099 & $15.019 \pm 0.067$ & $14.793 \pm 0.108$ & $14.650 \pm 0.151$ \\
V7 & 78.426274 & -65.458389 & $14.810 \pm 0.057$ & $14.521 \pm 0.081$ & $14.391 \pm 0.097$ \\
V8 & 78.428394 & -65.455498 & $14.981 \pm 0.064$ & $14.687 \pm 0.080$ & $14.637 \pm 0.143$ \\
\hline
\end{tabular}

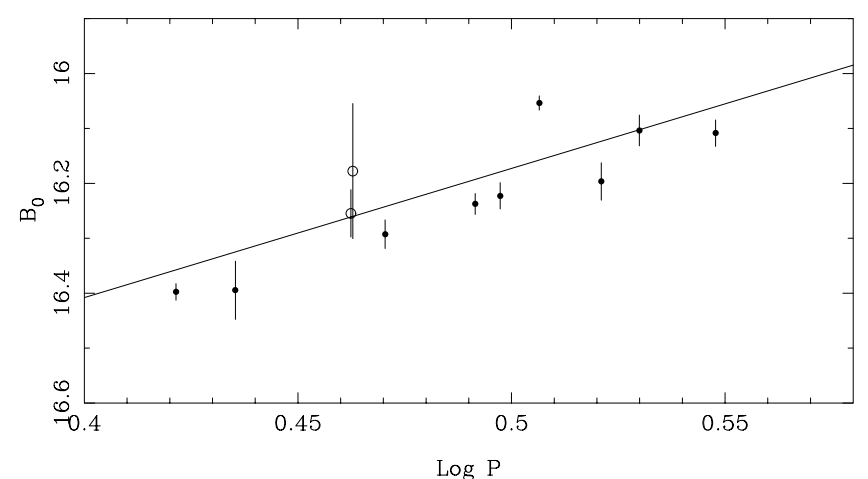

Fig. 3. $P L$ relation in the $B$-band, for the adopted slope of -2.352 and $E(B-V)=0.12$. The open circles represent the two overtone pulsators, plotted at their fundamental period.

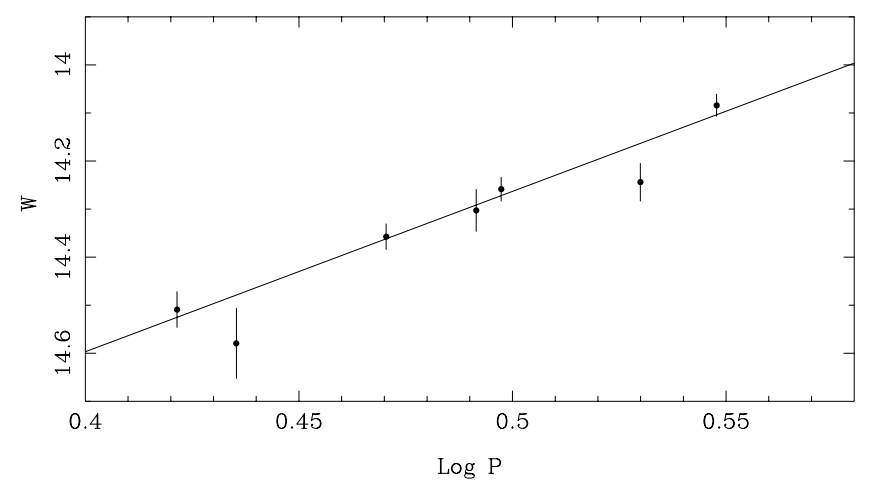

Fig. 4. $P L$ relation for the Wesenheit index, for the adopted slope of -3.337 .

for their associated large error. Fouqué et al. (2003) provide an alternative calibration for the Galactic Cepheids slopes, based on surface brightness methods: $-3.57 \pm 0.10$ in $W,-3.24 \pm$ 0.11 in $I,-2.72 \pm 0.12$ in $B$, and $-3.06 \pm 0.11$ in $V$; again, the slopes in $B, V$ and $I$ are different than in case of the LMC field Cepheids, and are also different from the NGC 1866 results at more than $1 \sigma$ level. The result that Galactic Cepheids have a different $P L$ slopes compared to the LMC ones contradicts the standard assumption of universality of the $P L$ relationship; if confirmed, this occurrence allows one to firmly exclude a solar

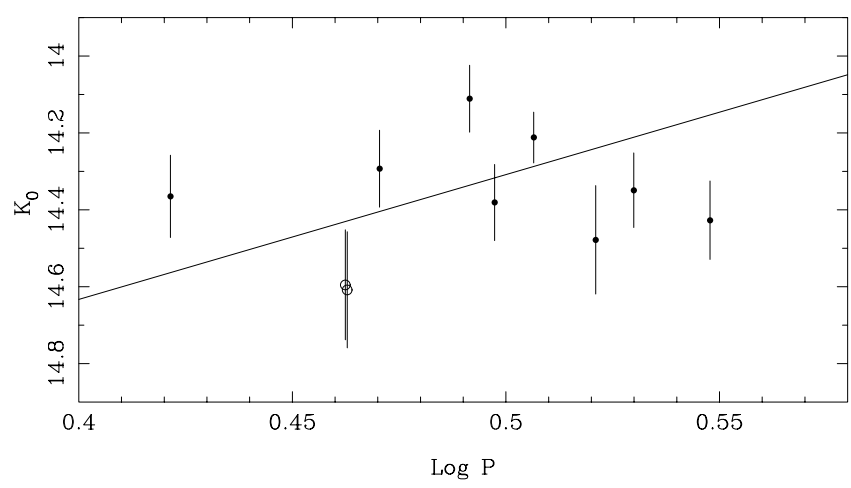

Fig. 5. $P L$ relation in the $K$-band, for the adopted slope of -3.246 and $E(B-V)=0.12$. The open circles represent the two overtone pulsators, plotted at their fundamental period.

metallicity for NGC 1866 (one of the possible explanations for the distance discrepancy mentioned in S03).

As for the $P L$ relationship in $K$ (Fig. 5) the data are very much scattered, and do not allow a meaningful determination of the $P L$ slope in this photometric band.

The second step in our analysis has been the determination of the distance of NGC 1866 from the LMC main body by using Cepheid stars. Due to the statistical agreement between the $P L$ slopes (in $W, I, B$ and $V$ ) for the cluster and the field, and the similarity between the spectroscopic $[\mathrm{Fe} / \mathrm{H}]$ determination for the cluster $-[\mathrm{Fe} / \mathrm{H}]=-0.5 \pm 0.1$ according to Hill et al. (2000) - and the mean $[\mathrm{Fe} / \mathrm{H}]$ of LMC Cepheids and supergiants $-[\mathrm{Fe} / \mathrm{H}] \sim-0.4$ according to Luck \& Lambert (1992) we can safely assume for the cluster Cepheids the very accurate slopes obtained for the field LMC objects. First, we fitted the $W P L$ relationship for the field LMC Cepheids to the cluster objects; this relationship is reddening independent, therefore we do not have to use any information regarding the cluster $E(B-V)$. Adopting the ZP for the LMC field Cepheids from Groenewegen (2000), we obtained a relative distance modulus of $0.04 \pm 0.03 \mathrm{mag}$ with respect to the LMC one, implying that the cluster is slightly more distant than the LMC main body. In order to establish a connection between this result and the distance between the cluster and its surrounding LMC field, one needs a model for the geometry of the LMC. There is a general consensus about the fact that the LMC is a disk galaxy with an 
Table 5. The distance between the field around NGC 1866 and the LMC centre.

\begin{tabular}{cccc}
\hline \hline$\theta$ & $i$ & Reference & $\Delta$ \\
$\left(^{\circ}\right)$ & $\left(^{\circ}\right)$ & & $(\mathrm{kpc})$ \\
\hline 258 & 38 & Schmidt-Kaler \& Gochermann (1992) & 0.37 \\
258 & 33 & Feitzinger et al. (1977) & 0.33 \\
261 & 25 & Weinberg \& Nikolaev (2000) & 0.38 \\
296 & 18 & Groenewegen (2000) & 1.02 \\
232 & 29 & Martin et al. (1979) & -0.65 \\
212 & 35 & van der Marel \& Cioni (2001) & -1.61 \\
\hline
\end{tabular}

approximately planar geometry; the two basic parameters to be evaluated are the inclination angle $i$ and the position angle $\theta$ of the line of nodes (the intersection of the galaxy plane and the sky plane), for which different estimates exist in the literature, as reported in Table 5. These different values for $i$ and $\theta$ imply different distances between the field around NGC 1866 and the LMC centre, as displayed in Table 5. For a distance modulus of $\sim 18.50 \mathrm{mag}$ to the galaxy centre, the distances reported in Table 5 correspond to a difference between -0.07 and +0.04 mag around this value. By interpreting the cluster distance modulus offset by $0.04 \pm 0.03$ mag with respect to the galaxy main body as the distance between NGC 1866 and the LMC centre, we obtain a difference of the distance moduli to the cluster and the surrounding field $\Delta(D M)$ ranging between zero and -0.11 mag (the field being closer), depending on the accepted values of $i$ and $\theta$. This result definitely rules out the possibility that the value $\Delta(D M)=+0.20 \pm 0.10$ obtained by $\mathrm{S} 03$ can be attributed to the cluster being closer.

At this point we can also redetermine the cluster reddening by imposing that the distances obtained from the $B, V$ and $I$ $P L$ relationships (which depend on the assumed cluster reddening) must provide the same relative distance from the LMC main body as obtained from $W$. We do not use the $K$-band to derive $E(B-V)$, due to the large spread of the cluster data in this $P L$ plane and its weak sensitivity to the reddening; however, we will employ $K$ data (with the $P L$ slope fixed by the LMC field Cepheids, as determined by Groenewegen 2000) as a sanity check for the results obtained from $V$ and $I$.

In our analysis we will use the following extinction ratios:

$$
\begin{aligned}
A_{B} & =4.32 E(B-V) \\
A_{V} & =3.24 E(B-V) \\
A_{I} & =1.96 E(B-V) \\
A_{K} & =0.35 E(B-V)
\end{aligned}
$$

following Schlegel et al. (1998), homogeneously with the OGLE-II (Udalski et al. 1999b) extinction maps; we recall that the zero point of the LMC PL relationships is derived from U99 data and the OGLE-II extinction maps, which provide an average reddening $E(B-V)=0.15$ for the LMC Cepheids.

By assuming the canonical value $E(B-V)=0.06$, the cluster results to be more distant than the LMC main body by $0.31 \mathrm{mag}$ in $B, 0.22 \mathrm{mag}$ in $V$ and $0.18 \mathrm{mag}$ in $I$. Obviously, this dependence on colour suggests an incorrect reddening, and in fact we obtain agreement between the distances in $B, V, I$ and $W$ only when the reddening is higher than the canonical value. More in detail, we compute various distances cluster-LMC main body, by fixing the reddening each time at a different value. For each reddening selection a distance with its associated $1 \sigma$ error is obtained. Typical errors obtained from the fitting procedure are $\pm 0.02 \mathrm{mag}$ in $I, \pm 0.05 \mathrm{mag}$ in $V$ and $\pm 0.07 \mathrm{mag}$ in $B$; we then enforce the condition that the "true" cluster reddening has to provide the same (within the respective error bars) relative distance in all of the three photometric bands, equal to the value obtained from the reddening free $W$ index. We obtain $E(B-V)=0.12 \pm 0.02$, where the error bar is basically determined by the more precise $I$-band data.

This is an important result, because it points out to a severe underestimate of the cluster reddening, with relevant implications for the MS-fitting distance. This reddening is also consistent (in the limit of the large dispersion of the $K$-band data) with the constraint imposed by the $K$-band data.

We have also performed, as a consistency check, a comparison between the positions of the cluster and field Cepheids in the $(V-I)_{0}-I_{0}$ Colour Magnitude Diagram (the $V$ - and $I$-band $P L$ relationships for the cluster Cepheids are the best defined ones), to verify if the cluster Cepheids are within the Colour Magnitude Diagram instability strip of the LMC field objects. Only FU field objects are displayed, since all cluster Cepheids with $I$ photometry available happen to be FU pulsators. We have used $E(B-V)=0.12$ for the cluster, the OGLE-II reddenings for the field Cepheids, and we have applied a correction of -0.04 mag to the $I$ magnitudes of the cluster objects, to account for their distance to the main body of the LMC derived before. Figure 6 displays the result of this comparison, and shows clearly that $E(B-V)=0.12$ for NGC 1866 is compatible with the location of the instability strip at the LMC metallicity.

As a final step we can try to derive an absolute value for the distance to NGC 1866 by applying a calibration for the absolute magnitude of the zero point of the Cepheids $P L$ relationship. The release of the Hipparcos database has prompted a calibration of the zero point based on parallaxes of Galactic Cepheids by means of the reduced parallax method (see, e.g., Feast \& Catchpole 1997; Groenewegen \& Oudmaijer 2000; Groenewegen 2000); the basic assumption in this calibration is that the slope of the Galactic $P L$ relationships are the same as in the LMC, where they can be accurately determined. The results by Tammann et al. (2003) and Fouqué et al. (2003) mentioned before seem to clearly point out to a dependence on metallicity, at least for the slope, whereas the situation about the zero point is not clear; therefore LMC distance estimates obtained with this kind of calibrations are affected by some uncertainty. Nevertheless, we applied the Groenewegen (2000) calibration of the absolute magnitude zero point of the reddening independent Wesenheit PL relationship to our NGC 1866 sample, obtaining a cluster distance modulus $D M=18.65 \pm 0.10$. Clearly, on the basis of the previous discussion, the reddening dependent distances obtained from the $V, I$ (and $K) P L$ relationships would provide a consistent result when $E(B-V)=0.12 \pm 0.02$ is assumed. Based on the geometrical corrections in Table 5, the 


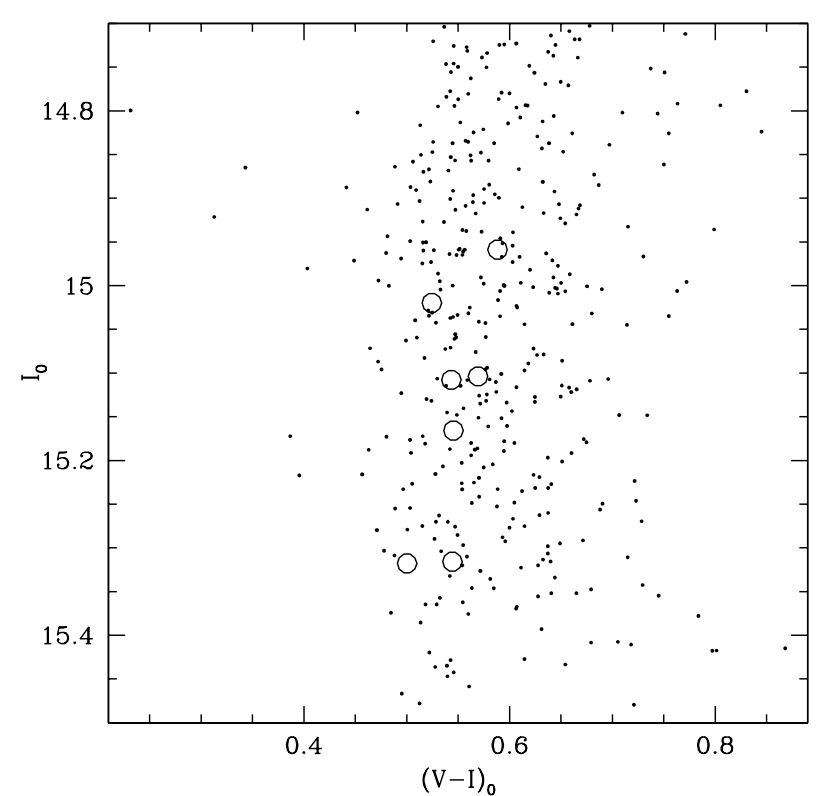

Fig. 6. $I_{0}-(V-I)_{0}$ diagram for the cluster (open circles) and FU LMC field (dots) Cepheids. A reddening $E(B-V)=0.12$ and a correction of -0.04 mag in $I$ have been applied to the cluster data. OGLE-II reddenings have been employed for the field Cepheids.

field surrounding NGC 1866 is located between $D M=18.65$ and $D M=18.54$. Notice how this last value agrees well with the RC distance estimated by $\mathrm{S} 03$.

\section{Discussion}

In the previous section we have obtained important results from the analysis of NGC 1866 Cepheid population. First, we have been able to derive a reddening independent relative distance between the cluster and the surrounding field, that goes in the opposite way with respect to what is necessary to explain the discrepancy found by S03. Cluster Cepheids provide a difference between the distance to the field around NGC 1866 and the distance to the cluster, $\Delta(D M)$, ranging between zero and -0.11 (depending on the exact geometry of the LMC disk), whereas S03 found $\Delta(D M)=+0.20 \pm 0.10$.

Second, from the observed $P L$ relationships in $V$ and $I$ we have obtained a new estimate of the cluster reddening, $E(B-V)=0.12 \pm 0.02$, which is on the same scale as the OGLE-II extinction maps of the LMC. This reddening is about twice the canonical value used for the cluster.

Third, in the assumption of universality of the Cepheid $P L$ relationships, we have obtained a cluster distance modulus $D M=18.65 \pm 0.10$. Based on the geometrical corrections reported in Table 5, this Cepheid distance implies that the field surrounding NGC 1866 is located at a distance between $D M=$ 18.65 and $D M=18.54$.

The new result about the cluster reddening has very important implications for the distance discrepancy discussed in S03; first of all, let us reexamine those results. The RC distance to the field surrounding NGC 1866 derived by $\mathrm{S} 03$ is $D M=$ $18.53 \pm 0.07$, and the simultaneous reddening determination provided $E(B-V)=0.05 \pm 0.02$. In a very recent paper
Salaris et al. (2003b) have studied in detail the systematic errors involved in the RC distance method, when one takes into account current uncertainties in the determination of the star formation history of the LMC, which is a crucial input parameter for applying the method. Based on Salaris et al. (2003b) results, one should revise the error bar on the previous estimate, obtaining $D M=18.53 \pm 0.07(\text { random })_{-0.05}^{+0.02}($ systematic $)$ and $E(B-V)=0.05 \pm 0.02$ (random) $)_{-0.04}^{+0.06}$ (systematic), where the systematic error is due to the uncertainty in the LMC star formation history. The MS-fitting distance to the cluster is $D M=$ $18.33 \pm 0.08$ when using a reddening $E(B-V)=0.064 \pm 0.011$ and the spectroscopic metallicity $[\mathrm{Fe} / \mathrm{H}]=-0.5 \pm 0.1$.

Our new determination of the cluster $E(B-V)$ provides a higher value, which implies a longer cluster distance from the MS-fitting technique, and therefore a potential solution to the distance problem found by S03. In light of the importance of this issue, we have reexamined the existing case for the canonical value $E(B-V)=0.06$ for the cluster.

The most direct empirical estimate of the cluster reddening before our analysis was based on photoelectric $U B V$ photometry of 4 stars observed by Walker (1974). The author compared the position of these 4 stars in the $(U-B)-(B-V)$ plane with a not clearly specified standard Pop I MS, and obtained (assuming $E(U-B)=0.72 E(B-V)$ ) a value quoted as $E(B-V)=0.061 \pm 0.0008$ averaging over the individual determinations made for the 4 stars ( 3 of them with 2 independent measurements, one with just one measurement); the formal error is extremely small, and it is possibly due only to the propagation of the internal error on the individual photometric data. However, the individual reddening estimates show a dispersion of $\sim 0.035 \mathrm{mag}$ around this mean value, which we believe is a better estimate of the error. Here, we have redetermined the cluster reddening (see Fig. 7) using the same method and cluster data, but using the standard MS Pop I sequence as reported in Table 3.9 of Binney \& Merrifield (1998). We have preliminarily checked two important effects. The first one is that the cluster Cepheids have metallicity lower than solar, the second one is that the observed 4 stars are most likely evolved off the Zero Age MS, whereas the standard Pop I sequence represents the Zero Age MS. In order to check the error introduced by these two factors, we used as a guideline the behaviour of theoretical isochrones (Girardi et al. 2000) of ages between 100 and $200 \mathrm{Myr}$ (the typical age of the cluster) in the $(U-B)-(B-V)$ plane, and found that both effects are practically negligible for the objects observed in NGC 1866. We then obtained from the colour-colour diagram a reddening $E(B-V)=0.08 \pm 0.03$, comparable with the Cepheid estimate. In addition, from the $(B-V)$ and $(U-B)$ colours of one blue MS star reported by Wa87, we obtained $E(B-V)=0.10 \pm 0.02$ by applying the same method.

The second evaluation used to support the canonical $E(B-V)=0.06$ was performed by van den Bergh \& Hagen (1968). They determined the reddening for a number of LMC clusters by comparing their integrated colours with a standard sequence of intrinsic colours of Galactic open clusters (based on results by Gray 1965; Schmidt-Kaler 1967), in the $(U-B)-(B-V)$ plane. They provide $E(B-V)=0.06$ for the cluster; the error bar on the individual estimate is unspecified 


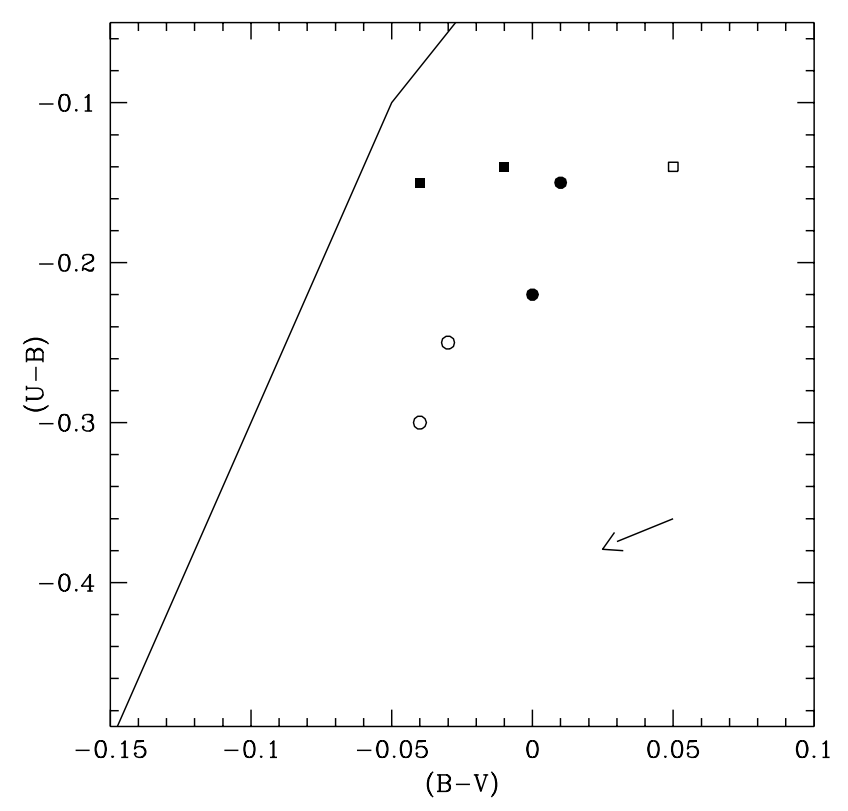

Fig. 7. $(U-B)-(B-V)$ diagram with the photometry of the 4 objects used by Walker (1974) to determine the cluster reddening. Different symbols correspond to different stars; 3 out of 4 stars have 2 observations. The solid line represents the Pop I standard Zero Age MS from Binney \& Merrifield (1998). The direction of the reddening vector is also displayed.

but they clearly state that "it should be emphasised that individual reddening values are quite uncertain”. Moreover, they did not take into account the effect of a possible metallicity difference between NGC 1866 and Galactic open clusters. We redetermined the cluster reddening using this same procedure, and both the colours provided by van den Bergh \& Hagen (1968) and the recent redetermination by Bica et al. (1996), which is $0.01 \mathrm{mag}$ bluer in $(B-V)$ and $0.04 \mathrm{mag}$ redder in $(U-B)$. We have used two alternative colour-colour standard sequences for the Galactic open clusters (see Fig. 8); the first one is the relationship given by Eq. (2) of Schmidt-Kaler (1967), which provides $E(B-V)=0.14 \pm 0.02$ and $E(B-V)=0.10 \pm 0.02$ from the van den Bergh \& Hagen (1968) and Bica et al. (1996) colours, respectively. In case of using the standard sequence reported in Eq. (6) of van den Bergh \& Hagen (1968), we obtain $E(B-V)=0.06 \pm 0.02$ and $E(B-V)=0.01 \pm 0.02$. The error bar on the individual determinations is due to the photometric error only, and not to the error associated to the determination of the standard sequences. It has been possible also to estimate the effect of the chemical composition, by using the theoretical results by Girardi et al. (1995). In the hypothesis that the Galactic standard sequence has solar metallicity, for a typical cluster age of $100 \mathrm{Myr}$ and $[\mathrm{Fe} / \mathrm{H}]=-0.5$ the estimates given above should be reduced by about 0.02 mag.

It is evident, on the base of this discussion, that the cluster integrated colours do not provide strong constraints on the cluster reddening due especially to the uncertainty in the standard Galactic sequence, and are not in clear contradiction with the reddening obtained from the Cepheids.

As a further check we have compared the cluster reddening with determinations of $E(B-V)$ for the surrounding field.

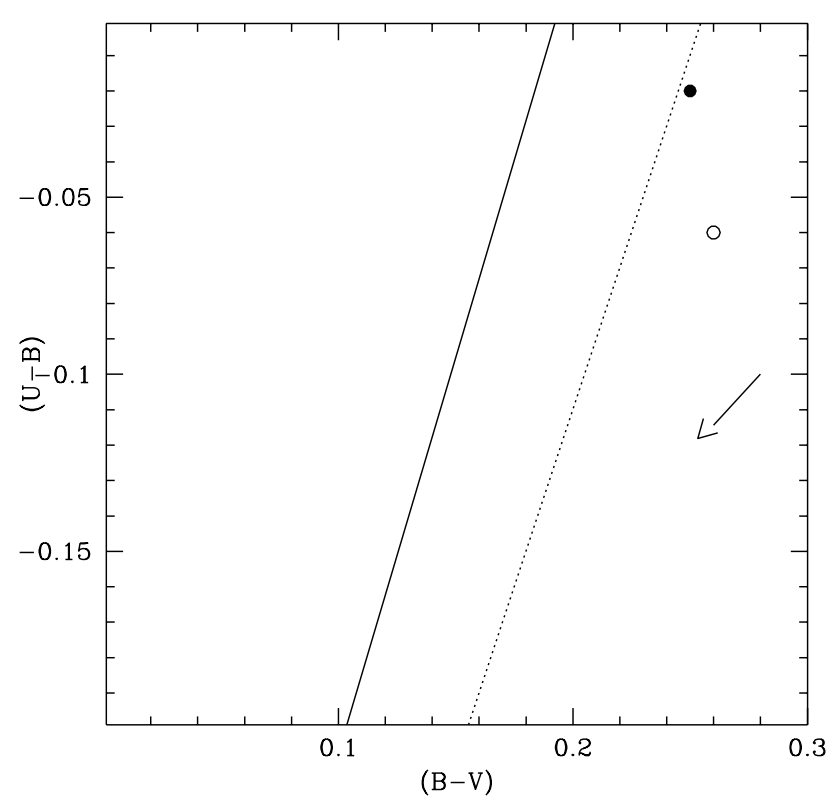

Fig. 8. $(U-B)-(B-V)$ diagram for NGC 1866. The filled circle represents the colours by Bica et al. (1996), the open circle the van den Bergh \& Hagen (1968) colours. The quoted photometric errors are equal to $0.02 \mathrm{mag}$ in $(B-V)$ and $(U-B)$ for Bica et al. (1996) data, $0.02 \mathrm{mag}$ in $(B-V)$ and $0.01 \mathrm{mag}$ in $(U-B)$ for van den Bergh \& Hagen (1968) data. The solid line represents the Pop I standard sequence by Schmidt-Kaler (1967), the dotted line the standard sequence used by van den Bergh \& Hagen (1968). The direction of the reddening vector is also displayed.

As discussed before, the multicolour RC method gives $E(B-$ $V)=0.05 \pm 0.02$ (random) ${ }_{-0.04}^{+0.06}$ (systematic) which, within the non negligible error bar, is not inconsistent with the Cepheid cluster value. We have in addition used the same OGLE-II technique to derive the reddening for the field around the cluster. The method is based on the assumption that the observed RC brightness in the I-band is constant in the LMC (at least for the bar-inner disk fields observed by OGLE-II) - due to similar Star Formation Histories - and therefore differences in its apparent $I$ magnitude correspond to extinction (hence reddening) differences when geometrical effects are negligible or accounted for. The zero point of the reddening (with an associated uncertainty by \pm 0.02 ) is fixed by other independent calibrators and confirmed by the recent work by Tammann et al. (2003). By comparing the RC apparent $I$ magnitude given by $\mathrm{S} 03$ for the field around the cluster, with the OGLE-II fields close to the LMC centre, we obtain $E(B-V)=0.11 \pm 0.02$. An additional systematic error by ${ }_{-0.020}^{+0.036}$ has to be added to this value, due to the uncertain geometric correction to the observed field, thus providing $E(B-V)=0.11_{-0.03}^{+0.04}$, consistent with the cluster reddening.

All this analysis is clearly based on the assumption that the extinction laws in the Galaxy and the LMC are the same in the $B, V, I$ wavelength range. It is not completely clear if there are differences for these photometric bands, but very recently Gordon et al. (2003) have published an average LMC extinction law which is slightly different from the Galactic one used in our study. More in detail, from Gordon et al. (2003) paper one obtains $A_{B}=4.41 E(B-V), A_{V}=3.41 E(B-V)$ 
and $A_{B}=2.14 E(B-V)$; with these ratios the Wesenheit reddening free index becomes $W=I-1.69(V-I)$ and one also finds that $E(U-B)=0.88 E(B-V)$. We have therefore redetermined the cluster reddening and the distance between the cluster and the LMC main body by using this LMC extinction law. Of course, this test overestimates the net effect of the LMC reddening law, because part of the extinction towards the cluster and the LMC field is due to the Galaxy (hence one should use the Galactic extinction law), and part to the LMC internal extinction.

First of all, we have considered the Wesenheit index, and obtained a cluster distance modulus relative to the LMC main body equal to $0.05 \pm 0.03 \mathrm{mag}$, almost the same value as for the case of using Galactic extinction ratios. We have then reestimated the cluster reddening from its Cepheid population by using the same procedure discussed in the previous section. As a first step we redetermined the zero points of the OGLE-II reddening maps using the Gordon et al. (2003) LMC extinction law; we found that the $E(B-V)$ zero points are changed by at most $0.01 \mathrm{mag}$ (in the direction of increased reddening). We then corrected appropriately the individual field Cepheid reddenings by considering a zero point $E(B-V)$ higher by $0.01 \mathrm{mag}$ and employing the LMC extinction ratios; we redetermined the field $P L$ relationships, that show an unchanged slope and zero points slightly brighter than what used in the previous chapter. These relationships have been then fitted to the cluster data (using again the above mentioned LMC reddening law) and, using the same procedure as before, we obtained a cluster reddening that is within $0.01 \mathrm{mag}$ of the value $0.12 \pm 0.02$ estimated before.

It appears therefore that changes in the extinction ratios consistent with available observations do not influence appreciably our determination of the reddening to the cluster. Also the estimates based on the colour-colour diagrams discussed before are not altered by more than $0.01 \mathrm{mag}$ when using these LMC extinction ratios.

We can now conclude by studying the effect of this new reddening determination on the MS-fitting distance modulus $^{2}$. When the Cepheid-based $E(B-V)=0.12 \pm 0.02$ is employed, and assuming the same spectroscopic metallicity $[\mathrm{Fe} / \mathrm{H}]=-0.5 \pm 0.1$ as in $\mathrm{S} 03$, the cluster $D M$ is increased by 0.25 mag with respect to $\mathrm{S} 03$ results, giving $D M=18.58 \pm$ 0.08 ; when compared to the RC distance of the surrounding LMC field, this distance provides $\Delta(D M)=-0.05 \pm$ 0.10 (random $)_{-0.05}^{+0.02}$ (systematic), in agreement with the values inferred from the Cepheids plus the geometrical corrections. The absolute values of the distance to the LMC obtained from both the RC method and the cluster MS-fitting are therefore consistent. It is interesting to notice that, within the $1 \sigma$ error bar, this MS-fitting distance is consistent with the result by Gieren et al. (2000b), based on the infrared surface brightness technique applied to one cluster Cepheid, which provides $D M=18.42 \pm 0.10$. This latter determination, albeit based at the moment on just one object, is largely insensitive

\footnotetext{
${ }^{2}$ Also these results are not affected appreciably if we use the LMC average extinction law by Gordon et al. (2003) instead of the Galactic one.
}

to uncertainties in the adopted reddening and extinction ratios, and therefore provides an independent check for the consistency of our reddening and distance estimates ${ }^{3}$.

Our revised MS-fitting cluster distance is also in agreement, within the corresponding $1 \sigma$ error bars, with the distance obtained from the Wesenheit $P L$ relationship applied to the cluster Cepheid population, in the assumption that it does not depend on the metallicity. By fixing the $P L$ slope to the value observed in the LMC and calibrating its zero point absolute magnitude on Galactic Cepheids with Hipparcos parallaxes (Groenewegen 2000), this $P L$ relationship provides $D M=$ $18.65 \pm 0.10$ for the cluster. This implies that within the period range spanned by the cluster Cepheids considered in our analysis, the metallicity effects on the Wesenheit $P L$ relationship appear to be small, and probably in the direction of slightly overestimating the cluster (which is on average more metal poor than local Cepheids) distance.

Acknowledgements. This research has made use of the SIMBAD database, operated at CDS, Strasbourg, France. This publication makes use of data products from the Two Micron All Sky Survey, which is a joint project of the University of Massachusetts and the Infrared Processing and Analysis Center/California Institute of Technology, funded by the National Aeronautics and Space Administration and the National Science Foundation. We thank an anonymous referee for his/her comments and suggestions, which improved the presentation of our results.

\section{References}

Alves, D., Rejkuba, M., Minniti, D., \& Cook, C. 2002, ApJ, 573, L51

Arp, H., \& Thackeray, A. D. 1967, ApJ, 149, 73 (AT67)

Benedict, G. F., McArthur, B. E., Fredrick, L. W., et al. 2002, AJ, 123, 473

Bica, E., Clariá, J. J., Dottori, H., Santos Jr., J. F. C., \& Piatti, A. 1996, ApJS, 102, 57

Binney, J., \& Merrifield, M. 1998, in Galactic Astronomy, Princeton Series in Astrophysics (Princeton University Press), 106

Côte, P., Welch, D. L., Mateo, M., Fischer, P., \& Madore, B. 1991, AJ, 101,1681

Cutri, R. M., Skrutskie, M. F., Van Dyk, S., et al. 2003, Explanatory Supplement to the 2MASS All Sky Data Release, http://www.ipac. caltech.edu/2mass/releases/allsky/ doc/explsup.html

Feitzinger, J. V., Schmidt-Kaler, T., \& Isserstedt, J. 1997, A\&A, 57, 265

Feast, M. W., \& Catchpole, R. M. 1997, MNRAS, 286, L1

Fouqué, P., \& Gieren, W. P. 1997, A\&A, 320, 799

Fouqué, P., Storm, J., \& Gieren, W. P. 2003, in Stellar candles for the extragalactic distance scale, Lecture Notes in Physics, ed. W. Gieren, \& D. Alloin (Springer), in press [astro-ph/0301291]

Freedman, W. 2001, ApJ, 553, 47

Gieren, W. P., Gómez, M., Storm, J., et al. 2000a, ApJS, 129, 111 (G2000)

Gieren, W. P., Richtler, T., \& Hilker, M. 1994, A\&A, 433, L73

${ }^{3}$ Gieren et al. (2000b) used a reddening of $E(B-V)=0.07$ in their analysis, and the $F_{V}-(V-K)$ surface-brightness relation of Fouqué $\&$ Gieren (1997). When using our derived reddening of $E(B-V)=$ 0.12 and the latest calibration by Nordgren et al. (2002) we estimate the surface-brightness based $D M$ will go up to 18.46 . 
Gieren, W. P., Storm, J., Fouqué, P., Mennickent, R. E., \& Gómez, M. 2000b, ApJ, 533, L107

Girardi, L., Chiosi, C., Bertelli, G., \& Bressan, A. 1995, A\&A, 298, 87

Girardi, L., Bressan, A., Bertelli, G., \& Chiosi, C. 2000, A\&AS, 141, 371

Gordon, K. D., Clayton, G. C., Misselt, K. A., Landoldt, A. U., \& Wolff, M. J. 2003, ApJ, 594, 279

Gray, D. F. 1965, AJ, 70, 362

Groenewegen, M. A. T. 2000, A\&A, 363, 901

Groenewegen, M. A. T., \& Oudmaijer, R. D. 2000, A\&A, 356, 849

Hill, V., Francois, P., Spite, M., Primas, F., \& Spite, F. 2000, A\&A, 364, L19

Luck, R. E., \& Lambert, D. L. 1992, ApJS, 79, 303

Martin, W. L., Warren, P. R., \& Feast, M. W. 1979, MNRAS, 188, 139

Nordgren, T. E, Lane, B. F., Hindsley, R. B., \& Kervella, P. 2002, AJ, 123,3386

Pietrzyński, G., \& Gieren, W. P. 2002, AJ, 124, 2633

Salaris, M., \& Girardi, L. 2002, MNRAS, 337, 332

Salaris, M., Percival, S., Brocato, E., Raimondo, G., \& Walker, A. R. 2003a, ApJ, 588, 801 (S03)

Salaris, M., Percival, S., \& Girardi, L. 2003b, MNRAS, in press [astro-ph/0307329]
Schmidt-Kaler, T. 1967, AJ, 72, 526

Schmidt-Kaler, T., \& Gochermann, J. 1992, in Variable Stars and Galaxies, ed. B. Warner, ASP Conf. Ser., 30, 203

Shapley, H., \& Nail, V. M. 1950, AJ, 55, 249

Sperl, M. 1998, Comm. Astroseismology, 111, 1

Storm, J., Andersen, J., Blecha, A., \& Walker, M. F. 1988, A\&A, 190, L18

Tammann, G. A., Sandage, A., \& Reindl, B. 2003, A\&A, 404, 423

Thackeray, A. D. 1951, MNRAS, 111, 206

Udalski, A., Szymański, M., Kubiak, M., et al. 1999a, AcA, 49, 201

Udalski, A., Soszyński, I., Szymański, M., et al. 1999b, AcA, 49, 223

van den Bergh, S., \& Hagen, G. L. 1968, AJ, 73, 569

van der Marel, R. P., \& Cioni, M.-R. L. 2001, AJ, 122, 1807

Walker, M. F. 1974, MNRAS, 169, 199

Walker, A. R. 1987, MNRAS, 225, 627 (Wa87)

Walker, A. R. 2003, in Stellar candles for the extragalactic distance scale, Lecture Notes in Physics, ed. W. Gieren, \& D. Alloin (Springer), in press [astro-ph/0303011]

Walker, A. R., Raimondo, G., Di Carlo, E., et al. 2002, ApJ, 560, L139

Weinberg, M. D., \& Nikolaev, S. 2001, ApJ, 548, 712

Welch, D. L., Mateo, M., Côte, P., Fischer, P., \& Madore, B. 1991, AJ, 101, 490 (We91) 